\title{
Verzeichnis der erstgenannten Autoren
}

Prof. Dr. G. M. Cochrane

GUY's Hospital, Department of Thoracic Medicine,

St. Thomas Street, London SE 19 RT

Prof. Dr. R. J. Ginsberg

Mount Sinai Hospital, Department of Thoracic Surgery, University Toronto, 600 University Avenue, Toronto M5 G15, SW 451 Ontario Canada

PD Dr. K.-J. Gräf

Abteilung für Innere Medizin, Medizinische Poliklinik, Universitätsklinikum Rudolf Virchow, Standort Charlottenburg, Spandauer Damm 130, 1000 Berlin 19

Prof. Dr. H. Fabel

Abteilung Pneumologie, Medizinische Klinik der Medizinischen Hochschule im Krankenhaus Oststadt, Podbielskistr. 380, 3000 Hannover 51

Prof. Dr. H. Matthys

Abteilung Pulmologie, Klinikum der Albert-Ludwigs-Universität Freiburg, Medizinische Klinik und Poliklinik, Hugstetter Str. 55, 7800 Freiburg i. B.

Prof. Dr. J. Meier-Sydow

Zentrum für Innere Medizin, Abteilung für Pneumologie, J.-W.-Goethe-Universität, Theodor-Stern-Kai 7, 6000 Frankfurt 70

Prof. Dr. B. A. Reitz

John Hopkins Hospital, Department of Surgery, 618 BlaLock Building, $600 \mathrm{~N}$ Wolfe Street, Baltimore MD21205, USA

Dr. B. Rothhut

Inserm U. 129, Unité de Recherches en Génétique et Pathologie Moléculaires, 24, Rue du Faubourg Saint-Jacques, 75674 Paris, Cedex 14

Prof. Dr. W. Schmutzler

Medizinische Fakultät der Rheinisch-Westfälischen Technischen Universität, Pharmakologie und Toxikologie, Reimser Str. 16, 5100 Aachen 
XII Verzeichnis der erstgenannten Autoren

Prof. Dr. J. P. Scott

Papworth Hospital, Papworth Everard, Cambridge CB3 8RE, UK.

Prof. Dr. U. Wahn

Universitäts-Kinderklinik und Polikinik, Klinikum Rudolf Virchow, Standort

Charlottenburg, Spandauer Damm 130, 1000 Berlin 19 